\title{
'SystemsX' - Project for a New Biological Science in Switzerland [1]
}

\author{
Gian-Reto Plattner*
}

\begin{abstract}
The understanding of a complex biological system requires an interdisciplinary approach; systems biology, wholly integrating various sciences including biology, physics, mathematics, informatics, chemistry, microelectronics, micromechatronics, as well as information-, bio- and nanotechnologies. Systems biology is a highly interactive scientific approach to biological research. It deals with the quantitative analysis of entire biological systems by investigating all components and networks of a given system. Dynamic computer models make it possible to simulate and change the properties of a cell, and potentially the whole organism. The general strategy of systems biology is the use of iterative cycles of experiment, modelling and prediction as they have been used successfully in the exact sciences such as physics and chemistry. If this approach succeeds in systems biology, this apparent paradigm change will transform today's biology from a predominantly descriptive science into an exact science. The Swiss Federal Institute of Technology, ETHZ, the Universities of Basel and Zürich as well as the research-oriented Swiss pharma industry stand together to develop systems biology in Switzerland. Their project named 'SystemsX' aims at obtaining worldwide competitive size as well as the role of a powerful global player. Economic and social needs provide the driving forces for entering this new, exciting area of research. Confederation, cantons, national research agencies and industry are the pillars for financing this major bridgehead from biology to the other disciplines.
\end{abstract}

Keywords: Interdisciplinary approach · Life sciences · New biology · Swiss Federal Institute of Technology (ETHZ) · Systems biology · 'SystemsX' • University of Basel · University of Zürich

\section{Introduction}

"The Swiss Federal Institute of Technology in Zürich (ETHZ), the University of Basel, the University of Zürich, the Cantons of Basel City, Basel Country and Zürich, and the Swiss Confederation together engage in a large project with the open and promising name 'Systems $X$ ' and thereby attain globally competitive size and the role of a powerful global player."

"Economic and social needs are the drivers for entering this new exciting area of research. Confederation, Cantons, na-

\footnotetext{
$\overline{{ }^{*} \text { Correspondence: }}$ Prof. Dr. G.-R. Plattner Vice-Chancellor of the University of Basel Petersgraben 35

$\mathrm{CH}-4003$ Basel

Tel.: +41612672735

E-Mail: gian-reto.plattner@unibas.ch
}

tional institutions for the advancement of science as well as industry are the pillars for financing this major bridge to other scientific disciplines."

" 'Systems $X$ ' is aimed at uniting the existing as well as new potential in systems biology at the partner universities. Other institutions are invited to join the project."

These were the words chosen to describe the project 'SystemsX', which the presidents/rectors of the participating universities used when they submitted it at the beginning of 2004 to the Swiss University Conference asking for cofinancing. Project 'SystemsX' came into existence, as many unusual projects do, following an intricate path. It started bottom-up when a group of scientists from Basel floated proposals to set up a 'Basel Institute for the Diseases of Aging' (BIDA); however, they received little response and could not light the fire of enthusiasm. For a while, there was only some glow left in the ashes, yet the fire ignited again when the idea of involving the leading Swiss University, the ETHZ, and subsequently the University of Zürich set the fantasies of many important stakeholders in motion.
In the minds of the proponents involved, the idea of a life science research area, Northwestern Switzerland, took shape with the promise to overcome the Helvetic federal fragmentation by a powerful union, similar to the 'arc lémanique' in the French part of Switzerland. All of a sudden, a worldwide leading role in a promising area of research and development, systems biology, appeared to be within reach. The big pharma companies in Basel joined the bandwagon with significant mental engagement. Beside the scientific arguments, economic and national research interests became important, too. The vision received its present name 'SystemsX' and seized the imagination of the population at large.

The governments of Basel-Stadt and Basel Landschaft, both cantonal parliaments, the responsible federal minister, heads of the economy, the boards of the University of Basel, the ETHZ and the University of Zürich, and finally the Swiss University Conference all lined up explicitly behind 'SystemsX' within the span of two years.

Today, 'SystemsX' is still a vision; it will have to prove itself in international competition. However, the first three 
years of creation have been financed, the project organization has been set up, two scientific initiatives have been formulated and the first appointments of scientific top shots under the label 'SystemsX' are under way.

\section{2. 'SystemsX': Pathway to the New Science Systems Biology}

The participating universities and the other stakeholders see a unique chance in 'SystemsX'. Together they can reach the first milestone of a new biology in Europe. This is happening at the same time as six new centres in the USA are being conceived and built - well-timed with the beginning of the 21 st century, the 'century of biology'.

After 50 years of 'preliminary achievements', science now has a rough overview of the basic principles of living organisms, of the genome, the functional gene products and some of its regulating mechanisms. We also recognize the limitations of the present biology and have some idea of the potential of the unknown beyond these limits. For the exploration of these unknown areas, and last but not least for responsible ways and means in dealing with biological organisms, a new, comprehensive approach will be required, which goes way beyond the past classical research in biology.

Systems biology deals with the quantitative analysis of entire biological systems by investigating all components and networks of a given system. Dynamic computer models make it possible to simulate and change the properties of a cell, and potentially the whole organism. The general strategy of systems biology is the use of iterative cycles of experiment, modelling and prediction as they have been used successfully in the exact sciences such as physics and chemistry. If this approach succeeds in systems biology, today's biology will transform from a predominantly descriptive into an exact science.

The paradigm change to 'systems biology', to a new quantitative systematic science und technique, still has to be elaborated by integrating elements of biology, physics, mathematics, informatics, chemistry, microelectronics and micromechatronics. Information-, bio-, nanotechnologies and their merger are looked upon as the key technologies of the 21 st century. The expectations regarding these technologies include revolutionary insights and new applications, such as the observation and variability of biological molecules, manufacturing of biochips for fast diagnosis, miniaturized sensors to measure immune parameters, early warning systems etc.

"Systems Biology is a highly interactive scientific approach to biological research. It requires and is dependent on different fields of expertise, including biology, engineering, computer science, mathematics, chemistry, etc. The fact that these areas of expertise are not usually present in a single research group is the main reason for the need to create a collaborative and interactive environment."

"It takes a cross-disciplinary faculty biologists, computer scientists, chemists, engineers, mathematicians, and physicists - who speak and understand the languages of these different disciplines to facilitate the development of new global technologies and to integrate these with the data acquisition, storage, integration, and analysis tools of computational biology and mathematics. A major challenge is to give the technologists a deep understanding of biology and vice versa."

"In addition, the technologists, computer scientists, and biologists must share a common language. This requires new approaches to describing and teaching biology. Together these technologies must be integrated with biology and medicine." (Ruedi Aebersold, Institute of Systems Biology, Seattle)

Thus, systems biology is not a segment of classical biology, but a new, independent discipline, not only affecting research, but including teaching, and, considering its application potential, also of industry. The next generation of young researchers trained in systems biology will contribute to the innovation of existing companies and will lay the foundations for new enterprises.

\section{Comprehensive Approach and New Forms of Organisations}

Comprehensive approaches are called for, on all levels of size and time scales, in many systems in nature, environment and technology such as weather and climate, communication, medicine, energy, or economic cycles. A current example is the development of miniaturized biocompatible microelectronic systems on which, in the same laboratory, experts from component physics, manufacturing technology, material science, switching design, informatics, chemistry and biology work together. Each discipline is indispensable for the success of the project. Beside the competence of the individual scientists, team building and effective communication are decisive for success.

The study of complex systems is the real reason for the interdisciplinary approach. Central is the research topic or the development target, e.g. the biosystem to be explored scientifically or to be realized by engineering. Around this target, the scientists from the required disciplines are called upon to reach the aim (Fig. 1). Modern sciences and technologies can afford this working style. However, adequate forms of organization still have to be tested and optimized constituting a nice and necessary challenge to scientific-technical problem solving.

\section{Systems Biology in the International Context}

International biology is engaging in the new field of systems biology with great drive. The first international conference on systems biology took place in Tokyo in 2000. The second congress was held in Heidelberg in 2004. In the USA, Japan, and Canada as well as in many European countries, several research teams have established themselves dealing with partial as-

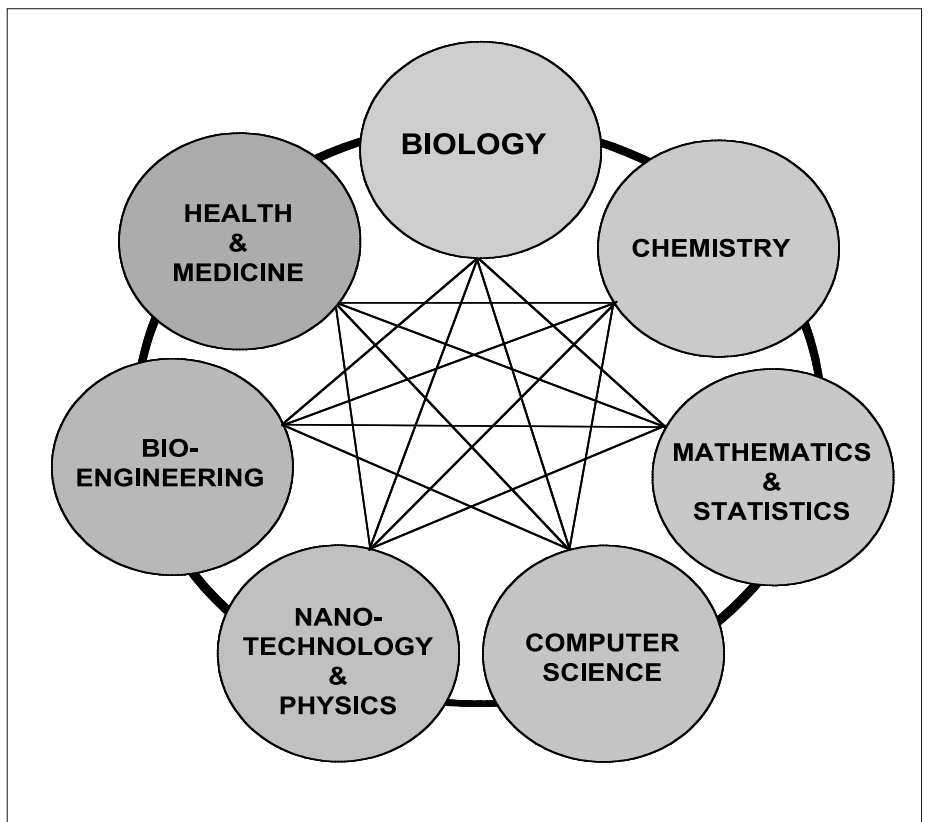

Fig. 1. The understanding of a complex biological system requires an interdisciplinary approach wholly integrating various sciences including biology, physics, mathematics, informatics, chemistry, microelectronics, micromechatronics, as well as information-, bio- and nanotechnologies. This apparent paradigm change transforms biology into an exact science. 
pects of systems biology. However, the necessary interdisciplinary integration and comprehensive approach have only been realized in few institutions.

Recently, various start-ups of major units in the US (Seattle, Harvard, Berkeley, Dallas, MIT, Stanford, California, Cornell) und Japan (Sony Systems Biology Group) have taken place. New curricula in new departments offer students from the very beginning lectures in biology, nanotechnology, computer sciences and electronics. National research programs offer financing specifically for this research area. In most cases, analysis and modelling of metabolic networks are at the core of these efforts.

\section{5. 'SystemsX' as a Framework for Systems Biology in Zürich and Basel}

ETHZ, the Universities of Basel and Zürich as well as the research-oriented Swiss pharma industry - within the scope of common research projects - have committed to do research in the area of systems biology. Their project named 'SystemsX' aims at obtaining worldwide competitive size as well as the role of a powerful global player. Economic and social needs form the driving forces for entering this new, exciting area of research. Confederation, cantons, national research agencies and industry are the pillars for financing this major bridgehead from biology to the other disciplines.

In the project 'SystemsX' the available as well as new potential in systems biology will be united at the partner universities. Other institutions can join the project. The project management consists of the president of ETHZ as chairman, the rectors of the Universities of Basel and Zürich, respectively, the officials responsible for research at these three universities as well as the heads of research of Novartis and F. Hoffmann-La Roche Inc (Roche).

The three participating universities support their existing and planned research teams in organizing themselves into interdisciplinary and scientifically oriented variable clusters and to work on common problems in the area of systems biology. These bottom-up interdisciplinary groups and clusters, which may be located at different sites, can identify and realize common research targets, define new needs for expertise as well as elaborate new teaching concepts and programs to develop and use core facilities. Following the dynamics of interest, such groups should form quickly, and also dissolve quickly when priorities change. When supported by 'SystemX', they have to justify their financing. In such fluid and scientifically motivated groups, the involvement of external partners is un- problematic. This provides the basis for a fundamentally new organizational structure (Fig. 2) which abandons the existing structures of departments and faculties to meet the needs of the new systems biology approach.

The close collaboration of the partner universities in the area of systems biology will therefore not only result in a harmonized planning of the appointment of faculty members, but also in the establishment of common research projects, teaching programs, seminars and graduate schools including master- and $\mathrm{PhD}$ programs.

'SystemsX' includes different components at the ETHZ and at the Universities of Basel and Zürich which will be coordinated in mutual agreement, but without limiting their autonomies, Existing components include, for instance, the Functional Genomics Centre Zürich and the Glycomics Initiative (ETHZ and University of Zürich), Project Oncology Cell Transfer (University of Zürich), Bioinformatics-Initiative (Biocenter Basel-SIB-Novartis-Roche) as well as the Life Science Training Facility (genome analysis und training at the University of Basel). A new project proposed by ETHZ scientists will be directed at the systems biology-oriented approach to the investigation of metabolic diseases.

\section{First Specific Initiatives within the Framework of 'SystemsX'}

Apart from the continuing development of the specific strengths of the three universities, two initiatives will be pursued with special emphasis and additional finances within 'SystemsX' to catalytically realize the scientific paradigm change.

\subsection{C-BSSE in Basel \\ The New 'Centre of Biosystems Science and Engineering' of the ETH Zürich}

The ETHZ will build a 'Centre of Biosystems Science and Engineering' C-BSSE in Basel as an autonomous centre of excellence with its own resources including staff positions, space and finances. Offices, laboratories and coworkers will be in the C-BSSE, but its faculty will be retained by the ETHZ, affiliated with a department of their own choice and paid by C-BSSE. In the case of double professorships simultaneously at a department of the Universities of Basel, Zürich or possibly even at another Swiss university, the search committees will be interdisciplinary and composed of members from different universities.

Project management for the C-BSSE during the build-up phase (2004-2007) will be composed of a project leader (Acting Chairman) and a project team of professors from the three partner universities, appointed ad personam according to their competences. The project team will be supported by a project staff. On the basis of the research concept to be defined by the project leaders, it is expected that the first faculty positions will be advertised before the end of 2004. Suitable buildings with adequate laboratory infrastructure are available in Basel and can be used immediately.

The scientific direction of the C-BSSE follows the principle of optimal innovation and complementarity with existing foci at the partner universities. In parallel, research groups already active in areas in or close to systems biology in Basel, including the university and its hospital, Novartis, Roche but also C-BSSE, will be included in an interdisciplinary cluster. This will reinforce the existing tight connections between titular professors among industry researchers, research teams of university professors at the Friedrich-Miescher-Institute, common use of expensive infrastructures such as the Life Science Training Facility and the highpower computer system, as well as focussed project collaborations with strong industry participation. In particular, pending appointments will depend on the requirements of 'SystemsX' or C-BSSE, respectively. Until 2007, five full professorships will be open at the Biocenter whose main selection criteria will be their integration into 'SystemsX'.

\subsection{CLU-BSS in Zürich The 'Cluster of Biosystems Science' of the ETHZ und the University of Zürich}

CLU-BSS will, in particular, cover the fields of proteomics und transcriptomics with an emphasis on tumour research. Professor Dr. Ruedi Aebersold, arriving from the Institute of Systems Biology, Seattle on November 1 2004, will head the CLU-BSS. Different model systems will be employed in experiments conducted at the cluster.

ETHZ and the University of Zürich offer financial incentives to research groups motivated to push interdisciplinary research, emphasizing new areas of science. Incentives are e.g. additional doctoral and postdoctoral positions. Support can also mean that the university makes space available for limited temporary integration of different research groups.

Further appointments will complement the cluster. At the ETHZ, until 2007 there are eight faculty positions to fill in the context of systems biology, at the University of Zürich there will be ten openings. Again, one of the main criteria choosing the candidates will be their integration into 'SystemsX'.

Part of the financial means granted by the Swiss University Conference will be allocated to the University of Zürich for setting up a competitive support system for in- 


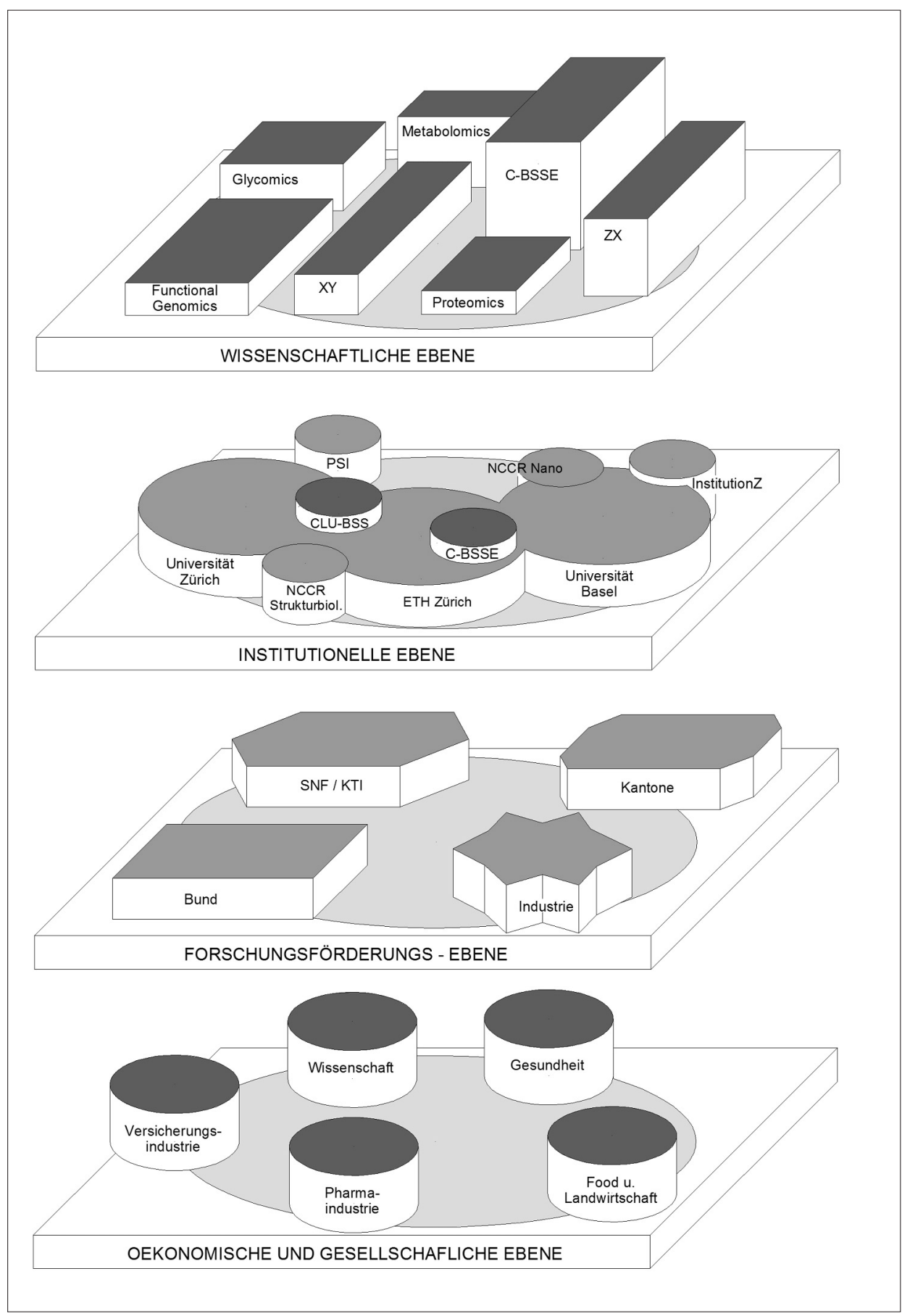

Fig. 2. Schematic position of project 'SystemsX' on the different levels. The two initiatives 'Centre of Biosystems Science and Engineering (C-BSSE) in Basel' and 'Cluster of Biosystems Science (CLUBSS) in Zürich' form the start of this new venture.

novative research and teaching in the area of systems biology, in collaboration with the partner universities. At the ETHZ such projects can already be supported by an internal support system.

\section{Finances 2004-2007}

The global financial means for the activities planned within the context of 'SystemsX', including money provided by the participating universities, amount to over CHF 100 million per year from 2004 to
2004-2007 agreed between the Federal Government and the ETHZ. For the period after 2008, the C-BSSE with 20 professors and 200-300 employees requires an annual budget of CHF 40 million.

Arrangements concerning construction, installation and rental costs for the centre C-BSSE of the ETHZ in Basel will be specified in a separate written agreement between the cantons of Basel Stadt and Basel Landschaft and the ETHZ.

Existing activities of the University of Basel in the area of proteomics/genomics and bioinformatics will run up to CHF 17 million by the end of 2007. In addition to the six faculty members already working in these domains, the University of Basel intends to fill openings at the Biocenter in a focussed manner. Additional CHF 2 million have been approved by the Swiss University Conference for respective activities at the University of Basel.

On the part of the ETHZ, five new full and three new assistant professorships are planned in systems biology in Zürich for the period 2004-2007. For these positions, for the financing of technology platforms and for research projects, investments of CHF 34 million are earmarked.

The University of Zürich plans to establish ten professorships in further areas in systems biology in the period 2004-2007. Towards this end, about CHF 30 million are budgeted during this time span. The Swiss University Conference approved additional CHF 3 million for activities at the University of Zürich in connection with CLU-BSS.

\section{Synergy Potentials in the Scientific and Economic Area Zürich - Basel}

The entire concept and the potential of the 'SystemsX' approach comprises much more than even the cumulated efforts of the three universities could achieve. In particular, the new centre C-BSSE will complement and stimulate the area of systems biology at the universities and in the research environment of the Basel pharma industry; its aim being to understand and demonstrate quantitatively fundamental biological principles. Only if a comprehensive qualitative and quantitative understanding of the processes and interactions in a system are on hand, can predictions be made about the behaviour at extreme external or internal influences or disturbances, respectively. Insights such as this are of high significance for the development of biomarkers and eventually for preventative medicine as well as for a differentiated health system.

Sharing work and responsibilities in research between C-BSSE und CLU-BSS or between the existing and expanding systems biology components at the three uni- 
versities does not follow a disciplinary scheme - e.g. bioinformatics in Basel, functional genomics in Zürich - but occurs spontaneously according to the different research objects and problems - e.g. focussing on simple or complex organisms or on different organs and diseases, respectively. It is essential that all components of systems biology in Zürich and Basel have critical mass and sufficient breadth from the beginning. Structures that concentrate one given discipline of project 'SystemsX' at one site, e.g. technology development in Basel or in Zürich, would offer little chance for success. The best bets for scientific success reside in having the researchers of the various disciplines work near one another or in a well functioning interdisciplinary network.

\subsection{University Site Zürich}

The two universities in Zürich have numerous internationally renowned research teams in the life sciences. The basic areas important for systems biology such as functional genomics, proteomics, pharmacogenomics, bioinformatics, biochemistry, structure biology, neuro sciences, cell biology, biotechnology, microbiology, immunology, biomedical technique, bioengineering, technical informatics, material sciences, clinical research, etc. are complementary and strongly represented in both universities in Zürich. The intense collaboration between the Zürich universities in the life sciences is reflected by the numerous double professorships, the double institutes, the joint NCCRs, several shared competence centres and the $\mathrm{PhD}$ programs. The Bologna reform aims at achieving a high coordination between the biology curricula of both universities. Various core facilities such as the Functional Genomics Center Zürich and the Imaging Center are already run jointly.

\subsection{University Site Basel}

The specific advantages of the Basel location lie in the close neighbourhood of university and industry with their corresponding cooperation potential. Basic research is being conducted at university institutes - Biocenter, Pharmacenter, NCCR nanosciences, Department Clinical-Biological Research (DKBW) - as well as at the industry funded Friedrich-Miescher-Institute (FMI). For a number of years, the Universities of Basel and Zürich and the ETHZ have established common research and teaching programs (ZH-BS Plant Science Centre, Pharmacentre Basel - Zürich). The University of Basel and ETHZ are members of the Swiss Institute for Bioinformatics and by this association tied to the universities of the Romandie, the French part of Switzerland.

Proteomics, functional genomics, bioinformatics, neurobiology, growth and development of cells to organs, infection biology, pharmacogenomics, oncology, immunology, biophysics, and imaging processes are core disciplines. The members of all academic research institutes already have tight relations among each other and to high-class teams at Novartis and Roche. Topics include common use of proteomic platforms, elaboration of genome databanks, etc. An important connecting function to Swiss nanotechnology is provided by the National Research Center (NCCR) nanosciences, whose Leading House is the Department of Physics at the University of Basel.

In addition, there are numerous biotechnology spin-offs in the trinational upper Rhine area.

\subsection{Pharma Site Basel}

The pharmaceutical industry of Basel has a strong interest in intensifying the collaboration between their own in-house and university research. Since 1996, industry has taken into consideration the consequences of the genomic era. Gene-mapping information will be freely available to al researchers as a basic tool. Modern medicine should be equipped with tools that allow diseases to be diagnosed before the appearance of symptoms und to apply intervention measures before damage becomes apparent. Towards this end, a comprehensive understanding of the qualitative and quantitative events and processes in cells are mandatory. Many target proteins can be formed by different pathways dubbed redundancy phenomenon. Without full understanding of the various pathways and their regulation mechanisms, it is hardly possible to develop efficient pharmaceuticals devoid of side effects. Trial-and-error would be the only - bad - solution.

Project 'SystemsX' and in particular the centre of excellence C-BSSE offers the opportunity to optimally shape the collaboration between the various basic research-oriented research groups in the area of systems biology at universities, in clinic and industry. The expansion and quantification of the understanding of biological systems and the focused, quantitative processing of ever larger masses of data from ever more precise experiments on ever more complex systems, necessary for this purpose, will be the challenge of the collaboration between the new C-BSSE and the research groups working in the systems biology environment at the ETHZ and the Universities of Basel and Zürich

At Roche and Novartis alone, over 2000 highly qualified scientists are working. Strong signals come from industry indicating that their own researchers - particularly in the area of systems biology - wish for a closer collaboration with basic research at the universities. Thus, this project is an eminent contribution to preserve or rather reinforce the scientific and industrial location Switzerland. Within Europe, the project 'SystemsX' could conquer the top spot immediately. The close collaboration with industry next door will create a unique combination which exists elsewhere only in Boston, San Diego und San Francisco.

\section{Conclusion}

'SystemsX' has been initiated, driven by the fervent passion of few, by the critical enthusiasm of some and by the joint scepticism of many scientists, public officials, research and industry managers. The boldness of its aims and conception is a fortunate exception in an increasingly hesitant Switzerland.

Many have joined forces to push this visionary project, although not every detail is clear and assured as yet and probably never will be.

It is the nature of the future that it is unsure and therefore harbours risks. He who risks nothing has no good future or one which he did not shape himself. 'SystemsX' offers a future which we can shape. If the stakeholders can lay aside the traditional parochialism, prioritize common interests, clear their mental hurdles and believe with confidence in success, then success there will be!

The proponents of 'BIDA', which stood at the beginning of the tangled ways of the evolution of 'SystemsX', added to their position paper of February 14, 2001, the following citation from Shakespeare's King Lear:

There is a tide in the affairs of men,

Which, taken at the flood, leads on to fortune....

On such a full sea are we now afloat,

And we must take the current when it serves,

Or lose our ventures.

We did take advantage of fortunate tides. They did not carry us to India, but afforded a voyage which led us to new, promising territories. These await settlement, to the benefit of those involved, of our country and of science.

Received: October 11, 2004

[1] This report had to be written on very short notice, forcing the author to rely extensively on a text presented to the Swiss University Conference in spring 2004 by O. Kübler, president of ETH Zürich, H. Weder, chancellor of the University of Zürich, and U. Gäbler, chancellor of the University of Basel. Their text was based on an exposé prepared in 2003 by a group of scientists, chaired by W. Gruissem, with the aim to define the scientific focus of 'SystemsX'. 